\title{
Tailored Gires-Tournois etalons as tunable dispersion slope compensators
}

\author{
Xuewen Shu and Ian Bennion \\ Photonics Research Group, Aston University, Aston Triangle, Birmingham B4 7ET, UK \\ John Mitchell and Kate Sugden \\ Indigo Photonics, Faraday Wharf, Aston Science Park, Birmingham B7 4BB, UK
}

Received November 20, 2003

\begin{abstract}
We present a novel concept of a tailored Gires-Tournois etalon structure and show that such a device is useful for the fabrication of a dispersion-slope compensator with an almost arbitrary dispersion profile and also with tunability in the dispersion slope. (C) 2004 Optical Society of America

OCIS codes: $060.2340,060.4510$.
\end{abstract}

Wavelength-division multiplexing is a key technology for expanding the capacity of optical fiber transmission systems. With increasing bit rates, chromatic dispersion compensation is becoming increasingly more critical for such systems. However, a mismatch between the dispersion slopes of the transmission and dispersion-compensating fibers results in only one wavelength that is perfectly compensated and in different amounts of residual dispersion on all the other channels. The effect of a dispersion slope on system performance increases as the operating bandwidth increases and also as the transmission distance and the bit rate increase. Moreover, the dispersion slope itself may also change as a result of optical path reconfiguration caused by network and environmental-temperature variation. Thus dispersion compensators with dispersion-slope tunability are desirable for dispersion-managed high-speed wavelength-division multiplexing systems. Although there have been some reports of dispersion-slope compensators ${ }^{1-3}$ (DSCs), most of them can compensate only for dispersion slopes with fixed values, ${ }^{1}$ or for a variable slope but only for a single channel. ${ }^{2}$ Only a few schemes have shown both multichannel compensation capability and tunability. ${ }^{3}$

Recently, Gires-Tournois etalons (GTEs) have aroused extensive interest in multichannel dispersion compensation and dispersion-slope compensation. ${ }^{4,5}$ For dispersion-slope compensation, both bulk-optic ${ }^{4}$ GTE and distributed GTE ${ }^{5}$ (DGTE) structures have been reported. Both of the demonstrated schemes are based on slightly mismatching the free spectral range of the GTEs. Although the reported GTE-based DSCs can compensate for a fixed dispersion slope and have additional tunability in dispersion, ${ }^{4,5}$ the dispersion slope cannot be adjusted after fabrication. In this Letter we extend the GTE concept to demonstrate a tailored GTE structure and show that such devices are useful for the fabrication of DSCs with almost arbitrary dispersion-slope profiles and also dispersion-slope tunability.

A Gires-Tournois interferometer is a special configuration of a Fabry-Perot etalon, which consists of a partially reflective mirror (first mirror) and a $100 \%$ reflective mirror (second mirror). Recently, distributed GTEs were developed that replace traditional mirrors with directly UV-written Bragg gratings in optical fiber. ${ }^{6}$ Traditional GTEs and DGTEs have similar properties, such as periodic oscillation structures in group delay and dispersion, which make them suitable for identical multichannel dispersion control. In a GTE the reflectivity of the first mirror-grating is the main determinant of the dispersion properties (such as group-delay amplitude and dispersion amplitude) of the structure. The concept proposed here involves a tailored GTE that is used to produce arbitrary group-delay profiles by tailoring the reflectivity profile of the first mirror-grating; the reflectivity of the second mirror-grating is kept close to $100 \%$ to achieve an all-pass function. It is difficult to tailor the reflection spectra of bulk optical mirrors, and thus it would be difficult to achieve such structures. However, using fiber Bragg gratings in the GTE structure makes the achievement of a tailored GTE much easier because the reflection spectra of fiber Bragg gratings can be readily tailored during fabrication to have almost arbitrary profiles.

To obtain a target-group delay profile, $\Delta \tau(\lambda)$ (i.e., an envelope of the group-delay amplitude), we determined that the reflection spectrum of the first mirrorgrating of the GTE, $R_{1}(\lambda)$, should be tailored to the following profile:

$$
R_{1}(\lambda)=\left(\left\{\left[\frac{2 T_{0}}{\Delta \tau(\lambda)}\right]^{2}\right\}^{1 / 2}+1-\frac{2 T_{0}}{\Delta \tau(\lambda)}\right)^{2}
$$

where $T_{0}=2 n d / c$ is the round-trip time in the cavity, $n$ is the mode effective index, $d$ is the cavity length, and $c$ is the speed of light in vacuum.

We fabricated both linearly and nonlinearly tailored DGTEs in the experiments. As examples, three of them are shown in Fig. 1. These DGTEs have typical lengths of $\sim 11 \mathrm{~mm}$ and photoinduced refractiveindex changes of $\sim 0.003$. The measured group-delay spectra, the envelopes of group-delay amplitudes, and their corresponding reflection spectra of the first 

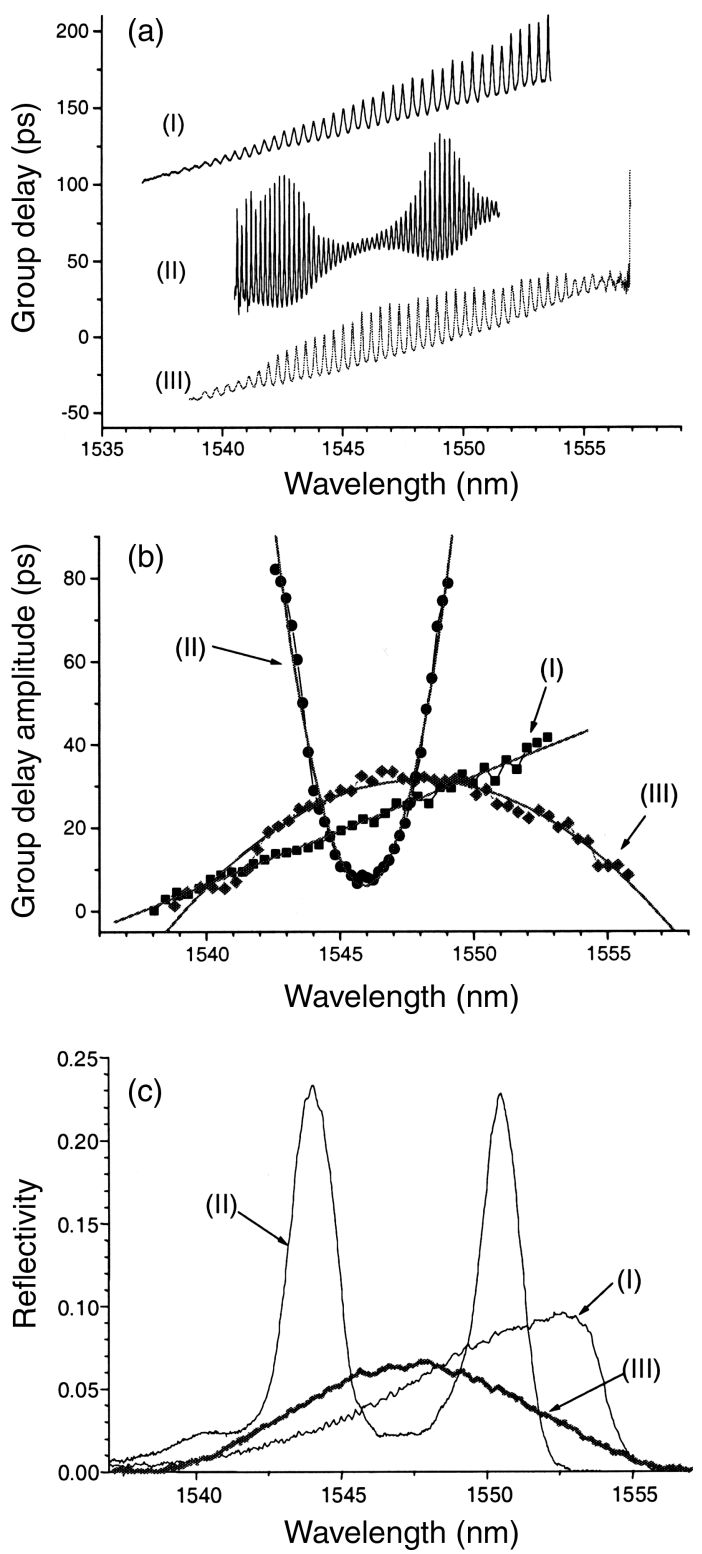

Fig. 1. Experimental examples of tailored DGTEs. (a) Measured group-delay spectra, (b) envelopes of group-delay amplitudes, (c) reflection spectra of the first grating recorded during the fabrication.

grating recorded during fabrication are plotted in Figs. 1(a), 1(b), and 1(c), respectively. It can be clearly seen from Figs. 1(a) and 1(b) that DGTE (I) has linearly increased amplitude in group delay, whereas DGTE (II) and DGTE (III) have positive quadratic and negative quadratic varied amplitudes, respectively, in group delay. Although the oscillation amplitudes of group delay vary channel by channel, for each channel the group delay is quadratic and results in linear variation in dispersion.

First we demonstrate a simple, tunable DSC achieved with a single linearly tailored DGTE. It is shown schematically in Fig. 2(a). Before tuning, each channel is set at zero dispersion; therefore the dispersion slope over these channels is zero. During tuning, different channels will suffer different dispersion changes $(\Delta \mathrm{D} 1<\Delta \mathrm{D} 2<\Delta \mathrm{D} 3)$; therefore a dispersion slope over these channels will be generated. The measured group-delay spectrum of the linearly tailored DGTE used in the experiment is shown in the top inset of Fig. 2(b). The different dispersion slope settings are made simply by application of different strains to the DGTE through a microdriver. (Adjustment by temperature control such as use of a thermoelectric cooler is preferred for practical applications.) The measured dispersion values for dispersion slope settings of $+23.8,+9.2,0.0,-10.8$, and $-23.4 \mathrm{ps} / \mathrm{nm}^{2}$ over $\sim 30$ channels are shown in Fig. 2(b), which clearly indicates slope tunability with such a tailored GTE. The drawback of this scheme is that the slope for each channel (internal) is not compensated; therefore such a DSC can be suitable only for a small-signal bandwidth. This shortcoming can be overcome by use of a pair of tailored DGTEs as described below.

The method of combining two tailored DGTEs to make a tunable DSC is shown schematically in Fig. 3(a). Both DGTEs have a linear envelope of group-delay amplitude over a certain number of channels. The envelopes are designed such that for each channel the dispersion slopes of the two DGTEs have the same magnitude but different signs in a specific region. Therefore for each channel there is a region where dispersion is constant when the two DGTEs are combined. When the dispersion for each channel is set at zero, as shown in Fig. 3(a), the dispersion slope



(a)

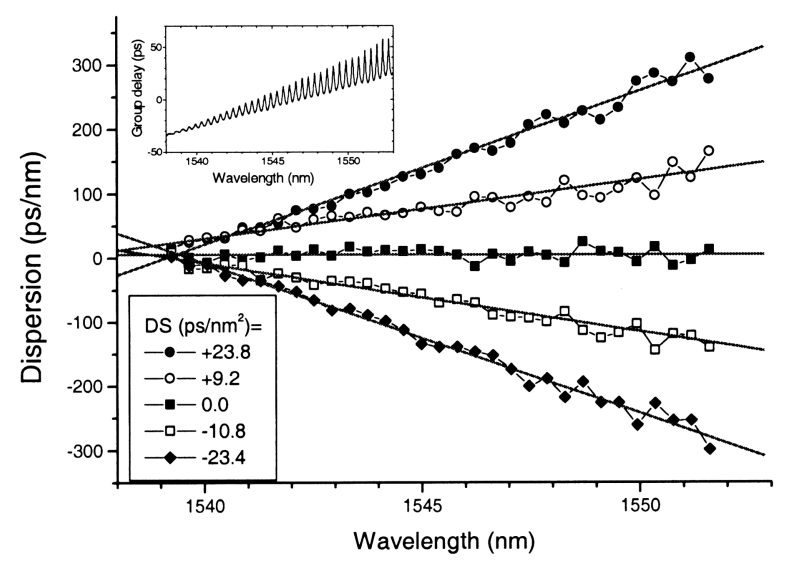

(b)

Fig. 2. Tunable DSC with a single linearly tailored DGTE. (a) Schematic of the setup, (b) measured dispersion values at several dispersion settings. Top inset, measured group-delay spectrum of the tailored DGTE. 


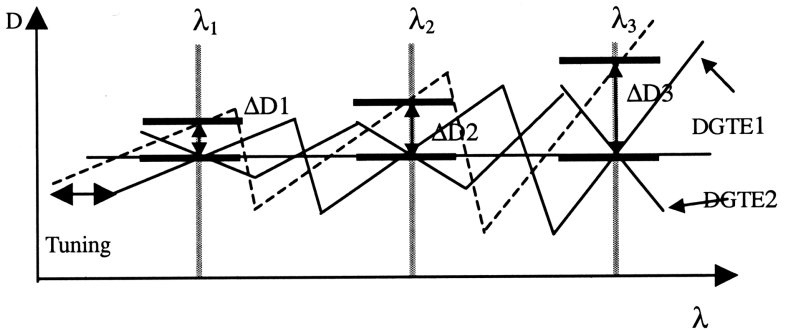

(a)

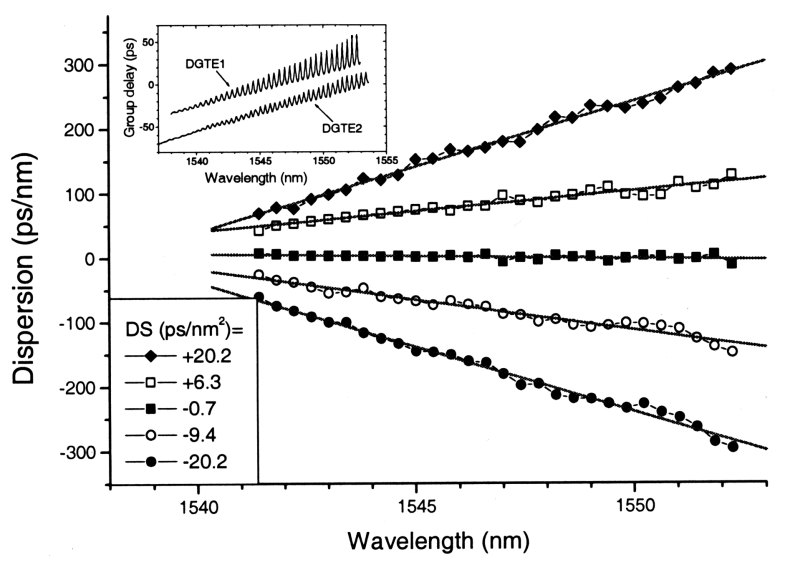

(b)

Fig. 3. Tunable DSC with two linearly tailored DGTEs (a) Schematic of the setup, (b) measured dispersion values at different dispersion settings. Top inset, measured group-delay spectra of the two tailored DGTEs.

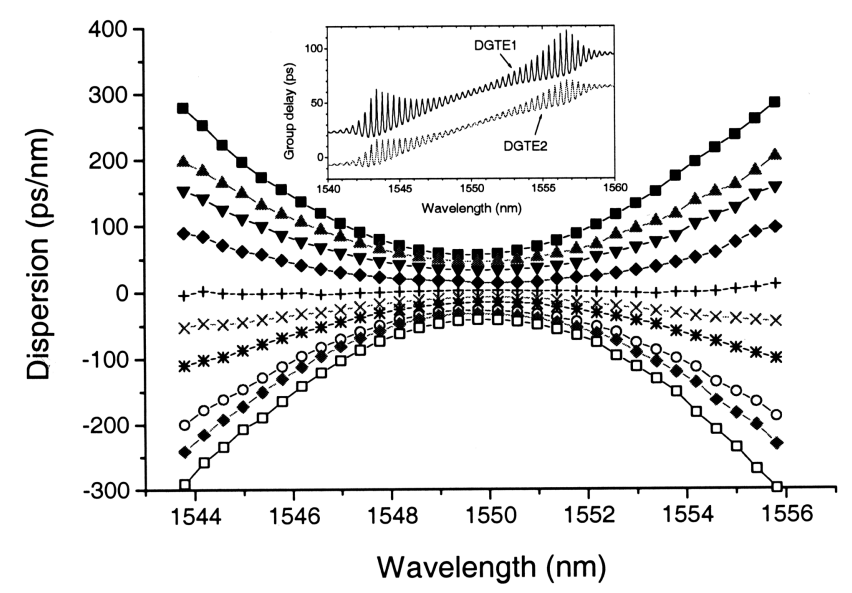

Fig. 4. Design of a tunable high-order DSC with two quadratically tailored DGTEs. Inset, calculated groupdelay spectra of the two tailored DGTEs.

over these channels is also zero. When the DGTE1 is tuned toward shorter wavelengths, the dispersions for all channels increase but the amounts of the dispersion changes are different, as shown in Fig. 3(a). Thus a dispersion slope is generated over these channels. The group-delay spectra of the two linearly tailored DGTEs selected for the experiment are shown in the inset of Fig. 3(b). The two DGTEs were combined by use of a four-port optical circulator. The measured results for dispersion slope settings of $+20.2,+6.3$, $-0.7,-9.4$, and $-20.2 \mathrm{ps} / \mathrm{nm}^{2}$ for nearly 30 channels are plotted in Fig. 3(b), which clearly indicates the slope tunability. The slope tuning range of this DSC is -22 to $+22 \mathrm{ps} / \mathrm{nm}^{2}$, and the usable bandwidth for each channel is $\sim 45 \%$ of the free spectral range $(50 \mathrm{GHz})$.

To demonstrate even higher-order dispersion compensation capability, we designed a DSC by using two quadratically tailored DGTEs. The simulated group-delay spectra for the two nonlinearly tailored DGTEs are shown in the inset of Fig. 4. The principle of tuning is similar to that in Fig. 3(a). When there is a relative spectral shift of the slopes, the higher-order slope (i.e., quadratic coefficients of the dispersion profile) will change. For the dispersion curves plotted in Fig. 4 the quadratic coefficients vary from +6.3 to -7.1 , and one can clearly see the change in the dispersion profile over these channels.

We have introduced the novel concept of a tailored Gires-Tournois etalon, which is an all-pass filter with a specially designed group-delay profile that we achieved by tailoring the reflectivity of the first mirror-grating. Linearly and nonlinearly tailored DGTEs have been fabricated with UV-written fiber Bragg gratings. We have successfully demonstrated the use of such devices as tunable dispersion-slope compensators that can compensate for complex dispersion profiles with tunability.

X. Shu's e-mail address is x.shu@aston.ac.uk.

\section{References}

1. K. Takiguchi, K. Okamoto, and T. Goh, Electron. Lett. 37, 701 (2001).

2. M. Jablonski, Y. Takushima, K. Kikuchi, Y. Tanaka, and N. Higashi, Electron. Lett. 36, 511 (2000).

3. Y. W. Song, Z. Pan, S. M. R. M. Nezam, C. Yu, Y. Wang, D. Starodubov, V. Grubsky, J. E. Rothenberg, J. Popelek, H. Li, Y. Li, R. Caldwell, R. Wilcox, and A. E. Willner, J. Lightwave Technol. 20, 2259 (2002).

4. D. J. Moss, M. Lamont, S. McLaughlin, G. Randall, P. Colbourne, S. Kiran, and C. A. Hulse, IEEE Photon. Technol. Lett. 15, 730 (2003).

5. X. Shu, K. Chisholm, and K. Sugden, presented at the 29th European Conference on Optical Communication, Rimini, Italy, September, 2003, paper We4.P.57.

6. X. Shu, K. Sugden, and K. Byron, Opt. Lett. 28, 881 (2003). 